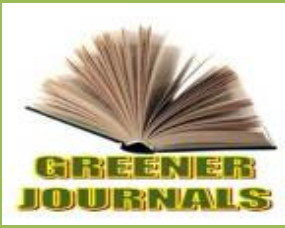

\title{
Growth, Yield and Elemental Status of Lycopersicum esculantum L. Grown in Fly ash with Azosprillum amended Soil
}

\section{Leela Veni ${ }^{1}$, Sabitri Nahak ${ }^{1}$ and Rajani Kanta Sahu ${ }^{2 \star}$}

\author{
${ }^{1}$ P.G. Department of Botany, Berhampur University, Berhampur-760007, Odisha, India \\ ${ }^{2}$ B.J.B Autonomous College, Bhubaneswar, Odisha, India
}

\section{ARTICLE INFO}

Article No.: 071215095

DOI: 10.15580/GJAS.2015.4.070715086

Submitted: $11 / 07 / 2015$

Accepted: 18/07/2015

Published: $31 / 07 / 2015$

${ }^{*}$ Corresponding Author

Rajani Kanta Sahu

E-mail: sahurajani.sahu@gmail. com

Keywords:

Flyash, Azosprillum, Growth,

Yield, Heavy metals,

Lycopersicum esculentum $L$
Fly ash (FA) from NALCO, Odisha (India) was used for amending soil at levels equivalent to $0,5,10,2040$ and $80 \mathrm{~T} / \mathrm{H}$ in which, tomato was grown and elemental residues of amended soil and plant were enumerated. In the present experiment Azosprillum mixed with graded levels of FA (5, 10, 2040 and $80 \mathrm{~T} / \mathrm{H})$ to observe the role of Azosprillum in improving soil quality and reducing metal toxicity. FA amendments caused significant improvement in soil quality and germination percentage of tomato plants. Growth (seedling height, Shoot length, leaf number) and yield (number of fruits, fruit weight and fruits per plant) of tomato increased with an increase in FA amendments. In the present study fly ash neutralize the pH imbalance, increased EC, CEC, OC, $P$ and some essential and nonessential micro elements i.e. $\mathrm{Fe}, \mathrm{Mn}, \mathrm{Pb}$, $\mathrm{Co}$ and $\mathrm{K}$. Further Azosprillum supplementations caused invariably increments in each growth parameters. The levels of 20 and $40 \mathrm{~T} / \mathrm{H} \mathrm{FA}$ were ideal for fruit yield. FA+ Azosprillum reduced heavy metal concentrations in both soil and plant. Hence, addition of bacterial species to FA may help in reducing the possible heavy metal toxicity of crop plants and can be used for solid waste management in Agro ecosystem. 


\section{INTRODUCTION}

Coal-fired power plants generate fly ash (FA) whose elemental composition (both nutrient and toxic elements) varies due to types and source of used coal (Camberato et al., 1997). In most instances FA consists of macronutrients, $\mathrm{Na}, \mathrm{K}, \mathrm{P}$, and $\mathrm{Fe}$ and micronutrients, $\mathrm{Co}, \mathrm{B}$, $\mathrm{Zn}, \mathrm{Cu}$, and $\mathrm{Mn}$. Heavy metals like $\mathrm{Pb}, \mathrm{Ni}, \mathrm{Cr}, \mathrm{Cd}$ and a few more, also occur abundantly and have the potential to cause contamination/toxicity (Fytianos et al., 2001). Further, plant micro-nutrients at high concentrations can cause metal toxicity (Miller et al., 2000). Although mobilized elements from dumped FA masses are potential sources of nutrients for plants there is a universal concern regarding heavy metal contaminations of terrestrial and aquatic ecosystems may also occur (Miller et al., 2000).

In India, about 80-million metric tons of FA is generated annually from thermal power stations with only a minor part used now for preparing bricks, ceramics and cements. Fly ash pond occupies an additional 100 ha land each year. Over flooding of fly ash water in rainy season to adjacent areas, like rice fields and other crop fields inevitably become contaminated, thus potentiating grave problems. Utilization of fly ash in agriculture is one of the option to reduce solid waste pollution. FA and other coal combustion byproducts have been used as nutrients supplements to crop soil in India and several countries (Miller et al., 2000; Mishra et al., 2009). Lower amendment levels of FA caused enhancements of both growth and yield while adverse effects at higher levels were observed for several crops including maize, soybean, barley, cabbage, apple, alfalfa, beet (Korcak, 1995; Miller et al., 2000). Further, higher levels of macro and micro-nutrients in FA have been shown to affect growth and yield of several crops in USA, Europe and India adversely, i.e., Maize and soybean (Mishra and Shukla, 1986), barley and cabbage (Koracak, 1995), apples (Fail and Wochok, 1977) and soybean (Allen et al., 1974). The present study aims to show the effect of FA alone and FA with Azosprillum on growth, yield and elemental status of Lycopersicum esculantum $\mathrm{L}$. in coasted lands of south Odisha, India.

\section{MATERIALS AND METHODS}

\section{Fly ash}

Electro-statically precipitated FA in an unweathered condition (Sample-lots less than 30 days old) obtained from NALCO power plant (Odisha) consists of the following (\%) by weight: sand 15.5 , silt 72.5 and clay 13 , with $\mathrm{pH} 7.4$ and the following elements (mg per $\mathrm{kg}$ ): $\mathrm{Na}$ 800, K 840, Fe 425, P 68, Ni 190, Co 670, Pb 200, Zn 340, Mn 450, Cu 700, Cr 505 and Cd 131, by analysis with an absorption spectrophotometer (Model AA 1475 at RRL, Bhubaneswar).

\section{Field preparation and Fly ash treatment}

The experimental fields were repeatedly ploughed to completely remove the rhizomes, roots of perennial grasses and shrubs. Then the field was left to be dried under sun for a week and then ploughed again. After 2-3 times thoroughly ploughed, experimental plots of $1 \times 1 \mathrm{~m}^{2}$ sizes were prepared. The experimental plots were uniformly spaced by 8 inch high and 1 feet wide ridge throughout. Soil samples were collected at a depth of 5$10 \mathrm{cms}$, air dried and stored for further analysis. All the experimental plots were mixed thoroughly with cow dung compost @ of 7.5T/H. Then the plots were amended with fly ash @ 5, 10, 20, 40, 80 TH 1kg of Azosprillum and mixed thoroughly by a spade. The fields were watered by sprinkling lightly so as to leave the soil with enough moisture for germination.

Disease free healthy tomato seeds were procured from the agriculture office, Jeypore (District: Koraput), Odisha, India. Five replicates of 100 seeds each were soaked overnight and next day sown in the fields. Sprouting was observed six days after sowing. Germination counts were recorded on each alternative day. 100 healthy seeds were selected from the soaked seeds. These seedlings were sown in the respective experimental plots with uniform spacing as per agronomic practice. All the experimental plots as well as the control plots were maintained alike.

\section{Soil analysis}

Soil pH: Soil of experimental plots was sandy loam, and $\mathrm{pH}$ of soil samples (without and with FA) was measured with a digital $\mathrm{pH}$ meter (Digison model, D1-707) in a soil/water mixture at the ratio of 1: 2.5 (Magdoff and Bartlett, 1985; Mishra et al., 2009).

Electrical conductivity (EC): Electrical conductivity (EC) expressed in $\mu \mathrm{Mho} / \mathrm{cm}$ of soil samples (FA/soil water at ratio of $1 / 5$ suspension) was determined following 30 min equilibration in a mechanical shaker, by a digital conductivity meter (Digison model, D1-909) (Khan and Khan, 1996).

Cation exchange capacity (CEC): Cation exchange capacity (CEC) of soil was determined by the methylene blue method; a lot of $2 \mathrm{~g}$ soil sample was equilibrated with $50 \mathrm{ml}$ of $1 \% \mathrm{NaCO}_{3}$, for titration against methylene blue as an indicator. The end point was noted by a light blue hallow circle a dark blue centre from a drop of solution on a Whatman no.1 filter paper; CEC value was determined by multiplying the titration factor with 0.535 and expressed as $\mathrm{C}$ mol. per $\mathrm{kg}$ (Sumner and Miller, 1996).

Organic Carbonic: Organic Carbonic values of 30days-old-soil samples were determined by oxidation with potassium dichromate in acid medium (Walkly and 
Black, 1934) to lots of $5 \mathrm{~g}$ of air-dried and sieved soil/FA samples, aliquots of $10 \mathrm{ml}$ of $1 \mathrm{~N} \mathrm{~K}_{2} \mathrm{Cr}_{2} \mathrm{O}_{7}$ solution, $20 \mathrm{ml}$ of $12 \mathrm{NH}_{2} \mathrm{SO}_{4}$ and $1.25 \% \mathrm{AgSO}_{4}$ were added with constant stirring. After incubation for $30 \mathrm{~min}$ to each sample a volume of $200 \mathrm{ml}$ distilled water was added followed by addition of $10 \mathrm{ml}$ phosphoric acid $(85 \%)$ and $1 \mathrm{ml}(0.42 \%)$. The indicator phenyl amine and titrated against $1 \mathrm{~N}$ ferrous ammonium Sulphate (Bremner and Jenkinson. 1960; Mishra et al., 2009).

Phosphorus 003A: The soil sample is treated with concentrated sulphuric acid and hydrogen peroxide to dissolve phosphorus in organic and non-silicate inorganic forms. Phosphorus in the silicate lattice is released by hydrofluoric acid treatment (Alef and Nannipieri, 1995).

Nitrogen: Discrete samples were made and pressed using washed sea sand and bentonite clay as a soil like matrix. Scotts Super Turf Builder $(36 \% \mathrm{~N})$ was added to the soil matrix for nitrogen samples. Measuring a total Nitrogen in soil was estimated by Using Laser-Induced Breakdown Spectroscopy (LIBS) (Harris et al., 2004).

Exchangeable Cations $\left(\mathrm{Na}^{+}, \mathrm{K}^{+}\right)$: Exchangeable cations are extracted from the soil using an extracting solution (1 N NH4OAc) at $\mathrm{pH}$ 7.0. The extracted solution is then analyzed by AA (atomic absorption) for the soil cations (Thomas et al., 1982).

\section{Elemental analysis}

Detailed methods of study of soil characteristics have been described previously (Harper et al., 1989). Digestion of soil samples were done in $20 \mathrm{ml}$ of mixed acids $\left(10 \mathrm{~N} \mathrm{HNO}_{3}, 12 \mathrm{~N} \mathrm{H}_{2} \mathrm{SO}_{4}, 60 \% \mathrm{HClO}_{4}\right.$ in the ratio of (5:0.5:1) (Shaw, 1992). Harvested leaves were thoroughly washed and oven-dried at $110^{\circ} \mathrm{C}$ for $40 \mathrm{~h}$, and the dried leaves ground to powders. Lots of the powders were digested with $10 \mathrm{~N} \mathrm{HNO}_{3}$ (Koracak, 1995) and subjected to elemental analysis using an atomic absorption spectrophotometer (Model AA1475, at Regional Research laboratory, Bhubaneswar), whereas $\mathrm{Na}$ and $\mathrm{K}$ contents were analyzed by flame photometry.

\section{RESULTS AND DISCUSSION}

Ex-situ germination patterns of tomato seeds were found to be better at 5 to $40 \mathrm{~T} / \mathrm{H}$ and the Azosprillum supplementations induced better germination pattern in 11 days study. Plant growth measured used as average seedling height, shoot height, leaf number, leaf area, flower number, number of fruits, fruit weight, and root length and plant dry weight. Clearly indicate that FA amendment significantly supported the growth of tomato (Table-1). The average seedling height increased from $3.7 \mathrm{~cm}$ (Control) to $3.8 \mathrm{~cm}(80 \mathrm{~T} / \mathrm{H} \mathrm{FA})$; the average shoot height increased from $30.37 \mathrm{~cm}$ (control) to $38.77 \mathrm{~cm}$ (80T/H FA); the average leaf number increased from 8.1 (Control) to $10.0(80 \mathrm{~T} / \mathrm{H} \mathrm{FA})$; the average number of flower increased from 11 (control) to 31 (80T/H FA); the average number of fruits increased from 7.2 (control) to $12.2(80 \mathrm{~T} / \mathrm{H} \mathrm{FA})$; the average fruit weight increased from $312.47 \mathrm{gm} / \mathrm{m}^{2}$ (control) to $429.12 \mathrm{gm} / \mathrm{m}^{2}$ (40 T/H FA); the average root length increased from $17.25 \mathrm{~cm}$ (control) to 22.11 (10 T/H FA); the average plant dry weight increased from $16.88 \mathrm{gm} / \mathrm{m}^{2}$ (control) to $25.27 \mathrm{gm} / \mathrm{m}^{2}$ (10 T/H FA). Azosprillum supplemented flyash perform better in all growth parameters (Table-1-9). The statistic analysis of data supported this conclusion. For levels of significance 2 types of comparisons are made, 1) between control and FA treatment (a), 2) between FA and FA+ Azosprillum (b). Levels of significance are indicated in respective tables.

Table 1: Effect of different concentrations of fly ash emended soil (without and with Azosprillum) on percentage of germination in Lycopersium esculentum C.V. S-28

\begin{tabular}{|c|c|c|c|c|c|c|c|c|c|c|c|c|}
\hline \multicolumn{13}{|c|}{ Days after sowing } \\
\hline \multirow{2}{*}{$\begin{array}{l}\text { FA Treatment } \\
\qquad(\mathrm{T} / \mathrm{H})\end{array}$} & \multicolumn{2}{|c|}{6} & \multicolumn{2}{|c|}{7} & \multicolumn{2}{|c|}{8} & \multicolumn{2}{|c|}{9} & \multicolumn{2}{|c|}{10} & \multicolumn{2}{|c|}{11} \\
\hline & FA & FA+ & FA & FA+ & FA & FAt & FA & FAt & FA & FAt & FA & FA+ \\
\hline Cont. & 35 & 26 & 39 & 41 & 40 & 47 & 40 & 45 & 40 & 47 & 41 & 47 \\
\hline 5 & 15 & 14 & 21 & 32 & 35 & 45 & 45 & 54 & 46 & 57 & 56 & 64 \\
\hline 10 & 29 & 13 & 30 & 57 & 41 & 61 & 49 & 64 & 57 & 64 & 61 & 65 \\
\hline 20 & 19 & 21 & 30 & 35 & 41 & 48 & 50 & 59 & 54 & 59 & 62 & 69 \\
\hline 40 & 20 & 17 & 32 & 44 & 40 & 49 & 46 & 49 & 58 & 61 & 63 & 73 \\
\hline 80 & 16 & 11 & 26 & 28 & 31 & 33 & 40 & 46 & 54 & 56 & 66 & 77 \\
\hline
\end{tabular}


Table 2: Effect of different concentrations of fly ash emended soil (without and with Azosprillum) on seedling height (in $\mathrm{cm}$ ) of Lycopersium esculentum C.V. S-28

\begin{tabular}{|c|c|c|c|}
\hline $\begin{array}{l}\text { FA Treatment } \\
(\mathrm{T} / \mathrm{H})\end{array}$ & $\begin{array}{l}\text { MEAN } \pm \text { SEM } \\
\text { Height (c.m.) }\end{array}$ & $\begin{array}{c}\text { FA+ } \\
\text { Treatment } \\
(\mathrm{T} / \mathrm{H})\end{array}$ & $\begin{array}{l}\text { MEAN } \pm \text { SEM } \\
\text { Height (c.m.) }\end{array}$ \\
\hline Cont. & $3.7 \pm 0.1^{\star \star \star}(\mathrm{a})$ & Cont & $3.19 \pm 0.8^{* \star *}(\mathrm{~b}) \mathrm{NS}(\mathrm{a}+)$ \\
\hline 5 & $2.60 \pm 0.1^{\star \star *}(\mathrm{a})$ & $5+$ & $2.60 \pm 0.1^{* \star *}(\mathrm{a}+) \mathrm{NS}(\mathrm{b})$ \\
\hline 10 & $2.50 \pm 0.2^{\star \star *}(\mathrm{a})$ & $10+$ & $2.50 \pm 0.2^{* * *}(a+)$ NS (b) \\
\hline 20 & $2.50 \pm 0.7^{\star \star *}(\mathrm{a})$ & $20+$ & $2.50 \pm 0.7^{\star * *}(\mathrm{a}+) \mathrm{NS}(\mathrm{b})$ \\
\hline 40 & $3.07 \pm 0.1 \mathrm{NS}(\mathrm{a})$ & $40+$ & $3.07 \pm 0.1^{\star * *}(\mathrm{a}+) \mathrm{NS}(\mathrm{b})$ \\
\hline 80 & $3.80 \pm 0.1 \mathrm{NS}(\mathrm{a})$ & $80+$ & $3.80 \pm 0.1^{* * *}(a+)$ NS (b) \\
\hline
\end{tabular}

Probability level: $\mathrm{p}<0.05,{ }^{* *} \mathrm{p}<0.01,{ }^{\star \star *} \mathrm{p}<0.001$, NS: Not Significant, $\mathrm{T} / \mathrm{H}$ : Tones per hectare $\mathrm{a}=$ Significant test between control and FA $a+=$ Significant test between control and FA+ $\mathrm{b}=$ Significant test between FA and FA+

Table 3: Effect of different concentrations of fly ash emended soil (without and with Azosprillum) on shoot length of Lycopersium esculentum C.V. S-28

\begin{tabular}{|c|c|c|c|c|c|c|c|c|}
\hline \multirow{3}{*}{$\begin{array}{c}\text { FA } \\
\text { Treatment } \\
(\mathrm{T} / \mathrm{H})\end{array}$} & \multicolumn{8}{|c|}{ Height (cm) } \\
\hline & \multicolumn{2}{|c|}{ 21DAS } & \multicolumn{2}{|c|}{ 31DAS } & \multicolumn{2}{|c|}{ 41DAS } & \multicolumn{2}{|c|}{ 51DAS } \\
\hline & FA & FA+ & FA & FA+ & FA & FA+ & FA & FA+ \\
\hline Cont. & $\begin{array}{c}5.95 \pm 0.15 \\
\text { NS(a) }\end{array}$ & $\begin{array}{c}5.5 \pm 0.25 \\
\star * \\
(\mathrm{~b}) \\
(\mathrm{a}+)\end{array}$ & $\begin{array}{l}9.82 \pm 0.28 \\
\mathrm{NS}(\mathrm{a})\end{array}$ & $\begin{array}{l}11.59 \pm 0.33 \\
* *(b)^{\star \star}(a+)\end{array}$ & $\begin{array}{c}16.25 \pm 0.43 \\
\text { NS(a) }\end{array}$ & $\begin{array}{c}17.08 \pm 0.4 \\
\mathrm{NS}(\mathrm{b})^{\star * \star} \\
(\mathrm{a}+)\end{array}$ & $\begin{array}{c}30.37 \pm 0.63 \\
*(a)\end{array}$ & $\begin{array}{c}32.94 \pm 0.52 \\
* * \star(b) N S(a+)\end{array}$ \\
\hline 5 & $\underset{* \star * \star}{5.9 \pm 0.13}$ & $\begin{array}{c}6.87 \pm 0.23 \\
* *(b) * * \\
(a+)\end{array}$ & $\begin{array}{l}9.78 \pm 0.27 \\
\text { NS(a) }\end{array}$ & $\begin{array}{l}13.39 \pm 0.29 \\
*(b) N S(a+)\end{array}$ & $\begin{array}{c}16.69 \pm 0.04 \\
\text { NS(a) }\end{array}$ & $\begin{array}{c}21.24 \pm 0.4 \\
* *(b) N S(a+)\end{array}$ & $\begin{array}{c}32.05 \pm 0.57 \\
* *(a)\end{array}$ & $\begin{array}{c}33.61 \pm 1.20 \\
* *(b) N S(a+)\end{array}$ \\
\hline 10 & $\begin{array}{c}7.49 \pm 0.25 \\
\star \star \star * \\
(a)\end{array}$ & $\begin{array}{c}6.9 \pm 0.14 \\
\mathrm{NS}(\mathrm{b})^{\star * *} \\
(\mathrm{a}+)\end{array}$ & $\begin{array}{c}10.27 \pm 0.43 \\
\mathrm{NS}(\mathrm{a})\end{array}$ & $\begin{array}{c}11.53 \pm 0.19 \\
\mathrm{NS}(\mathrm{b})^{\star \star \star}(\mathrm{a}+)\end{array}$ & $\begin{array}{c}16.39 \pm 0.32 \\
\text { NS(a) }\end{array}$ & $\begin{array}{c}17.9 \pm 0.33 \\
* \star(b) N S(a+)\end{array}$ & $\begin{array}{c}33.72 \pm 0.92 \\
\text { NS(a) }\end{array}$ & $\begin{array}{c}34.10 \pm 2.03 \\
N S(b){ }^{* *}(a+)\end{array}$ \\
\hline 20 & $\begin{array}{c}7.93 \pm 0.47 \\
\star \star \star \\
(\mathrm{a})\end{array}$ & $\begin{array}{c}7.73 \pm 0.27 \\
\mathrm{NS}(\mathrm{b})^{\star * *} \\
(\mathrm{a}+)\end{array}$ & $\begin{array}{c}11.39 \pm 0.59 \\
* * \star(a)\end{array}$ & $\begin{array}{c}12.39 \pm 0.24 \\
N S(b)^{\star * *}(a+)\end{array}$ & $\begin{array}{c}17.01 \pm 0.04 \\
\text { NS(a) }\end{array}$ & $\begin{array}{c}18.06 \pm 0.32 \\
N S(b) N S(a+)\end{array}$ & $\begin{array}{c}34.28 \pm 1.7 \\
* \star *(a)\end{array}$ & $\begin{array}{c}36.36 \pm 0.73 \\
N S(b)^{\star \star *}(a+)\end{array}$ \\
\hline 40 & $\begin{array}{r}7.09 \\
\pm 0.13 \\
\star \star \star \star \\
\end{array}$ & $\begin{array}{c}7.050 .12 \\
\mathrm{NS}(\mathrm{b})^{\star \star *} \\
(\mathrm{a}+)\end{array}$ & $\begin{array}{c}13.32 \pm 0.38 \\
* * \star \\
(a)\end{array}$ & $\begin{array}{c}13.32 \pm 0.38 \\
\star *(b){ }^{* \star *}(a+)\end{array}$ & $\begin{array}{c}17.23 \pm 0.42 \\
* * *(a)\end{array}$ & $\begin{array}{c}17.39 \pm 0.31 \\
* \star * \\
*(b){ }^{* \star \star}(a+)\end{array}$ & $\begin{array}{c}36.5 \pm 1.2 \\
* \star \star * \\
(a)\end{array}$ & $\begin{array}{c}36.84 \pm 0.93 \\
\star * \star *(b)^{\star * \star}(a+)\end{array}$ \\
\hline 80 & $\begin{array}{r}7.09 \\
\pm 0.13 \\
\end{array}$ & $8.17 \pm 0.26$ & $13.24 \pm 0.16$ & $14.2 \pm 0.29$ & $20.25 \pm 0.43$ & $21.50 \pm 0.47$ & $38.77 \pm 0.44$ & $42.05 \pm 0.72$ \\
\hline
\end{tabular}

Probability level: ${ }^{\star} \mathrm{p}<0.05,{ }^{* *} \mathrm{p}<0.01,{ }^{* * *} \mathrm{p}<0.001$, NS: Not significant, a: Significant test between flyash and control soil $\mathrm{T} / \mathrm{H}$ : Tones per hectare, DAS: Days after sowing $\mathrm{a}=$ Significant test between control and FA $\mathrm{a}+=$ Significant test between control and FA+ $\mathrm{b}=$ Significant test between FA and FA+ 
Table 4: Effect of different concentrations of fly ash emended soil (without and with Azosprillum) on number of leaves of Lycopersium esculentum C.V. S-28

\begin{tabular}{|c|c|c|c|c|c|c|}
\hline \multirow{3}{*}{$\begin{array}{c}\text { FA } \\
\text { Treatment } \\
(\mathrm{T} / \mathrm{H}) \\
\end{array}$} & \multicolumn{6}{|c|}{ Number of leaves (DAS) } \\
\hline & \multicolumn{2}{|c|}{23 DAS } & \multicolumn{2}{|c|}{33 DAS } & \multicolumn{2}{|c|}{43 DAS } \\
\hline & FA & FAt & FA & FA+ & FA & FA+ \\
\hline Cont. & $\begin{array}{c}4.6 \pm 0.16 \\
* \star \\
(\mathrm{a})\end{array}$ & $\begin{array}{c}5.8 \pm 0.19 \\
\star \star \star \\
(\mathrm{b}){ }^{*}(\mathrm{a}+)\end{array}$ & $\begin{array}{c}7.6 \pm 0.16 \\
* \star \star \\
(\mathrm{a})\end{array}$ & $\begin{array}{c}8.6 \pm 0.16 \\
\star \star(b)^{\star *}(a+)\end{array}$ & $\begin{array}{c}8.1 \pm 0.31 \\
* * \text { (a) }\end{array}$ & $\begin{array}{c}9.7 \pm 0.15 \\
\mathrm{NS}(\mathrm{b}) \mathrm{NS}(\mathrm{a}+)\end{array}$ \\
\hline 5 & $\begin{array}{c}5.5 \pm 0.22 \\
* \star * \\
(a)\end{array}$ & $\begin{array}{c}6.4 \pm 0.16 \\
\star \star \star \\
(b)^{\star \star \star}(a+)\end{array}$ & $\begin{array}{c}8.7 \pm 0.21 \\
*(a)\end{array}$ & $\begin{array}{c}7.9 \pm 0.17 \\
\star \star \\
(b)^{\star \star}(a+)\end{array}$ & $\begin{array}{c}10.0 \pm 0.51 \\
* \text { (a) }\end{array}$ & $\begin{array}{c}9.7 \pm 0.26 \\
*(b) N S(a+)\end{array}$ \\
\hline 10 & $\underset{* * * *(a)}{5.7 \pm 0.21}$ & $\begin{array}{c}7.4 \pm 0.16 \\
\star \star \star \\
(\mathrm{b})^{\star * \star}(\mathrm{a}+)\end{array}$ & $\begin{array}{c}8.3 \pm 0.21 \\
N S(a)\end{array}$ & $\begin{array}{c}9.2 \pm 0.12 \\
\mathrm{NS}(\mathrm{b})^{* * *}(\mathrm{a}+)\end{array}$ & $\begin{array}{c}9.7 \pm 0.63 \\
\mathrm{NS}(\mathrm{a})\end{array}$ & $\begin{array}{c}9.3 \pm 0.15 \\
N S(b) N S(a+)\end{array}$ \\
\hline 20 & $\begin{array}{c}5.6 \pm 0.16 \\
\star \star \star \star \\
(a)\end{array}$ & $\begin{array}{c}7.1 \pm 0.23 \\
* \star *(b)^{\star *}(a+)\end{array}$ & $\begin{array}{c}7.9 \pm 0.37 \\
\mathrm{NS}(\mathrm{a})\end{array}$ & $\begin{array}{c}7.3 \pm 0.15 \\
* *(b) N S(a+)\end{array}$ & $\begin{array}{c}9.3 \pm 0.71 \\
* \text { (a) }\end{array}$ & $\begin{array}{l}9.9 \pm 0.17 \\
* \star(b)^{*}(a+)\end{array}$ \\
\hline 40 & $\begin{array}{c}5.6 \pm 0.16 \\
\text { NS(a) }\end{array}$ & $\begin{array}{c}7.5 \pm 0.42 \\
* * *(b) N S(a+)\end{array}$ & $\begin{array}{c}7.4 \pm 0.22 \\
* \star \star \\
(a)\end{array}$ & $\begin{array}{c}8.7 \pm 0.26 \\
\mathrm{NS}(\mathrm{b})^{\star \star \star}(\mathrm{a}+)\end{array}$ & $\begin{array}{c}9.2 \pm 0.29 \\
\star \star \star \\
\text { (a) }\end{array}$ & $\begin{array}{c}10.4 \pm 0.22 \\
N S(b)^{\star *}(a+)\end{array}$ \\
\hline 80 & $4.7 \pm 0.15$ & $5.9 \pm 0.27$ & $6.6 \pm 0.16$ & $6.5 \pm 0.16$ & $10.0 \pm 0.25$ & $10.6 \pm 0.22$ \\
\hline
\end{tabular}

Probability level: ${ }^{*} p<0.05,{ }^{* *} p<0.01,{ }^{* *} p<0.001$, NS: Not significant, a: Significant test between flyash and control soil $\mathrm{T} / \mathrm{H}$ : Tones per hectare, DAS: Days after sowing $\mathrm{a}=$ Significant test between control and FA $\mathrm{a}+=$ Significant test between control and FA+ $\mathrm{b}=$ Significant test between FA and FA+

Table 5: Effect of different concentrations of fly ash emended soil (without and with Azosprillum) on number of flowers of Lycopersium esculentum C.V. S-28

\begin{tabular}{|c|c|c|c|c|c|c|c|c|c|c|c|c|c|c|c|c|}
\hline \multirow{3}{*}{$\begin{array}{l}\text { FA Treatment } \\
(\mathrm{T} / \mathrm{H})\end{array}$} & \multicolumn{16}{|c|}{ NUMBER OF FLOWERS (DAS) } \\
\hline & \multicolumn{2}{|c|}{48} & \multicolumn{2}{|c|}{50} & \multicolumn{2}{|c|}{52} & \multicolumn{2}{|c|}{54} & \multicolumn{2}{|c|}{56} & \multicolumn{2}{|c|}{58} & \multicolumn{2}{|c|}{60} & \multicolumn{2}{|c|}{62} \\
\hline & FA & FA+ & FA & FA+ & FA & FA+ & FA & FA+ & FA & FA+ & FA & FA+ & FA & FA+ & FA & FA+ \\
\hline Cont. & 0 & 0 & 0 & 0 & 0 & 0 & 0 & 0 & 0 & 0 & 0 & 0 & 0 & 7 & 11 & 29 \\
\hline 5 & 0 & 0 & 0 & 0 & 1 & 0 & 3 & 0 & 8 & 4 & 18 & 21 & 27 & 30 & 17 & 34 \\
\hline 10 & 1 & 0 & 0 & 3 & 2 & 4 & 2 & 3 & 5 & 10 & 16 & 23 & 22 & 33 & 23 & 31 \\
\hline 20 & 2 & 2 & 3 & 5 & 8 & 7 & 13 & 16 & 20 & 28 & 36 & 47 & 41 & 45 & 32 & 44 \\
\hline 40 & 0 & 6 & 1 & 7 & 3 & 12 & 10 & 24 & 25 & 30 & 42 & 51 & 43 & 62 & 47 & 50 \\
\hline 80 & 0 & 0 & 1 & 0 & 2 & 1 & 4 & 2 & 10 & 12 & 24 & 30 & 26 & 39 & 31 & 51 \\
\hline
\end{tabular}

FA: Flyash, T/H: Tones per hectare, DAS: Days after sowing $\mathrm{a}=$ Significant test between control and FA a+= Significant test between control and FA+ $\mathrm{b}=$ Significant test between FA and FA+

Table 6: Effect of different concentrations of fly ash emended soil (without and with Azosprillum) on number of fruits of Lycopersium esculentum C.V. S-28

\begin{tabular}{|c|c|c|c|c|c|c|}
\hline \multirow{3}{*}{$\begin{array}{c}\text { FA } \\
\text { Treatment } \\
(\mathrm{T} / \mathrm{H})\end{array}$} & \multicolumn{6}{|c|}{ Number of Fruits (DAS) } \\
\hline & \multicolumn{2}{|c|}{60} & \multicolumn{2}{|c|}{70} & \multicolumn{2}{|c|}{80} \\
\hline & FA & FA+ & FA & FA+ & FA & FA+ \\
\hline Cont. & $2.7 \pm 0.26 \mathrm{NS}(\mathrm{a})$ & $\begin{array}{c}2.7 \pm 0.33 \\
\mathrm{NS}(\mathrm{b})^{* * *}(\mathrm{a})\end{array}$ & $\begin{array}{c}5.7 \pm 0.85 \\
*(a)\end{array}$ & $\begin{array}{c}7.2 \pm 0.80 \\
\mathrm{NS}(\mathrm{b}) \mathrm{NS}(\mathrm{a}+)\end{array}$ & $7.2 \pm 0.59 \mathrm{NS}(\mathrm{a})$ & $\begin{array}{c}8.1 \pm 0.67 \\
* * *(b)^{* \star *}(a+)\end{array}$ \\
\hline 5 & $5.0 \pm 1.3 \mathrm{NS}(\mathrm{a})$ & $\begin{array}{c}5.3 \pm 0.51 \\
N S(b){ }^{* *}(a+)\end{array}$ & $10.0 \pm 0.14 \mathrm{NS}(\mathrm{a})$ & $\begin{array}{c}8.6 \pm 0.40 \\
\mathrm{NS}(\mathrm{b}) \mathrm{NS}(\mathrm{a}+)\end{array}$ & $8.2 \pm 0.48 \mathrm{NS}(\mathrm{a})$ & $\begin{array}{l}13.4 \pm 0.03 \\
* \text { (b) NS }(a+)\end{array}$ \\
\hline 10 & $4.0 \pm 0.76^{* \star *}(\mathrm{a})$ & $\begin{array}{c}5.6 \pm 0.68 \\
N S(b){ }^{* * *}(a+)\end{array}$ & $\begin{array}{c}7.5 \pm 0.93 \\
* \text { (a) }\end{array}$ & $\begin{array}{c}9.6 \pm 1.0 \\
N S(b){ }^{*}(a+)\end{array}$ & $7.4 \pm 0.47 \mathrm{NS}(\mathrm{a})$ & $\begin{array}{c}9.4 \pm 0.71 \\
* \star *(b){ }^{* \star *}(a+)\end{array}$ \\
\hline 20 & $8.7 \pm 1.5^{* * *}(\mathrm{a})$ & $\begin{array}{c}8.9 \pm 0.9 \\
*(b)^{* \star *}(a+)\end{array}$ & $9.2 \pm 1.2^{* * *}(\mathrm{a})$ & $\begin{array}{c}9.8 \pm 0.22 \\
N S(b) *(a+)\end{array}$ & $\begin{array}{c}6.9 \pm 0.43 \\
* *(a)\end{array}$ & $\begin{array}{c}11.9 \pm 0.7 \\
\mathrm{NS}(\mathrm{b})^{* * *}(\mathrm{a}+)\end{array}$ \\
\hline 40 & $7.8 \pm 0.94^{* * *}(a)$ & $\begin{array}{c}10.9 \pm 1.1 \\
\mathrm{NS}(\mathrm{b}){ }^{* * *}(\mathrm{a}+)\end{array}$ & $10.4 \pm 0.52^{* * *}(\mathrm{a})$ & $\begin{array}{c}10.3 \pm 1.1 \\
\mathrm{NS}(\mathrm{b}){ }^{*}(\mathrm{a}+)\end{array}$ & $\begin{array}{c}10.72 \pm 0.69 \\
\star \star \star \\
\text { (a) }\end{array}$ & $\begin{array}{c}12.18 \pm 0.51 \\
\mathrm{NS}(\mathrm{b})^{\star \star \star}(\mathrm{a}+)\end{array}$ \\
\hline 80 & $6.3 \pm 1.0$ & $7.9 \pm 0.75$ & $9.9 \pm 1.0$ & $9.8 \pm 0.62$ & $12.2 \pm 0.4$ & $13.94 \pm 0.64$ \\
\hline
\end{tabular}

Probability level: ${ }^{*}<0.05,{ }^{* *} \mathrm{p}<0.01,{ }^{\star \star *} \mathrm{p}<0.001$, NS: Not significant, a: Significant test between flyash and control soil

$\mathrm{T} / \mathrm{H}$ : Tones per hectare, $\mathrm{FA}$ : Flyash $\mathrm{a}=$ Significant test between control and FA $\mathrm{a}+=$ Significant test between control and $\mathrm{FA}+$ $\mathrm{b}=$ Significant test between $\mathrm{FA}$ and $\mathrm{FA}+$ 
Table 7: Effect of different concentrations of fly ash emended soil (without and with Azosprillum) on Fruit Weight/Plant $\left(\mathrm{gm} / \mathrm{m}^{2}\right)$ of Lycopersium esculentum C.V. S-28

\begin{tabular}{|c|c|c|c|}
\hline $\begin{array}{c}\text { FA } \\
\text { Treatment } \\
(\mathbf{T} / \mathbf{H})\end{array}$ & $\begin{array}{c}\text { Fruit } \\
\text { Weight/Plant } \\
\left(\mathbf{g m} / \mathbf{m}^{\mathbf{2}}\right)\end{array}$ & $\begin{array}{c}\text { FA+ } \\
\text { Treatment } \\
(\mathbf{T} / \mathbf{H})\end{array}$ & $\begin{array}{c}\text { Fruit } \\
\text { Weight/Plant } \\
\left(\mathbf{g m} / \mathbf{m}^{\mathbf{2}}\right)\end{array}$ \\
\hline Cont. & 312.47 & Cont.+ & 368.012 \\
\hline 5 & 418.29 & $5+$ & 433.7 \\
\hline 10 & 426.3 & $10+$ & 448.59 \\
\hline 20 & 429.12 & $20+$ & 458.63 \\
\hline 40 & 419.85 & $40+$ & 421.95 \\
\hline 80 & 362.16 & $80+$ & 384.33 \\
\hline
\end{tabular}

FA: Flyash, $\mathrm{T} / \mathrm{H}$ : Tones per hectare, DAS: Days after sowing $a=$ Significant test between control and FA

$a+=$ Significant test between control and $F A+$ $\mathrm{b}=$ Significant test between FA and FA+

Table 8: Effect of different concentrations of fly ash emended soil (without and with Azosprillum) on Root length of Lycopersium esculentum C.V. S-28

\begin{tabular}{|c|c|c|c|}
\hline $\begin{array}{c}\text { FA } \\
\text { Treatment } \\
(\mathbf{T} / \mathbf{H})\end{array}$ & Root length(cm) & $\begin{array}{c}\text { FA+ } \\
\text { Treatment } \\
(\mathbf{T} / \mathbf{H})\end{array}$ & Root length(cm) \\
\hline Cont. & $17.25 \pm 1.8 \mathrm{NS}(\mathrm{a})$ & Cont.+ & $21.3 \pm 0.87 \mathrm{NS}(\mathrm{b}) \mathrm{NS}(\mathrm{a})$ \\
\hline 5 & $22.11 \pm 2.3^{\star}(\mathrm{a})$ & $5+$ & $24.62 \pm 1.8^{\star * *}(\mathrm{~b})^{\star * *}(\mathrm{a})$ \\
\hline 10 & $22.11 \pm 0.92 \mathrm{NS}(\mathrm{a})$ & $10+$ & $33.52 \pm 2.8 \mathrm{NS}(\mathrm{b})^{* \star *}(\mathrm{a}+)$ \\
\hline 20 & $17.27 \pm 1.7 \mathrm{NS}(\mathrm{a})$ & $20+$ & $17.41 \pm 0.38 \mathrm{NS}(\mathrm{b})^{\star * *}(\mathrm{a}+)$ \\
\hline 40 & $15.69 \pm 1.6 \mathrm{NS}(\mathrm{a})$ & $40+$ & $17.25 \pm 0.47 \mathrm{NS}(\mathrm{b})^{* * *}(\mathrm{a}+)$ \\
\hline 80 & $15.89 \pm 1.1$ & $80+$ & $15.89 \pm 0.40$ \\
\hline
\end{tabular}

Probability level:: $\mathrm{p}<0.05,{ }^{* \star} \mathrm{p}<0.01,{ }^{* \star *} \mathrm{p}<0.001 \mathrm{NS}$ : Not significant, a: Significant test between flyash and control soil $\mathrm{T} / \mathrm{H}$ : Tones per hectare, FA: Flyash, DAS: Days after sowing

$\mathrm{a}=$ Significant test between control and FA

$\mathrm{a}+=$ Significant test between control and $\mathrm{FA}+$

$\mathrm{b}=$ Significant test between FA and FA+

Table 9: Effect of different concentrations of fly ash emended (without and with Azosprillum) on Plant Dry Weight of Lycopersium esculentum C.V. S-28

\begin{tabular}{|c|c|c|c|}
\hline $\begin{array}{c}\text { FA } \\
\text { Treatment } \\
(\mathrm{T} / \mathrm{H})\end{array}$ & $\begin{array}{c}\text { Dry Weight/Plant } \\
\left(\mathrm{gm} / \mathrm{m}^{2}\right)\end{array}$ & $\begin{array}{c}\text { FA+ } \\
\text { Treatment } \\
(\mathrm{T} / \mathrm{H})\end{array}$ & Dry Weight/Plant $\left(\mathrm{gm} / \mathrm{m}^{2}\right)$ \\
\hline Cont. & $16.88 \pm 1.0 \mathrm{NS}(\mathrm{a})$ & Cont.+ & $19.36 \pm 1.4 \mathrm{NS}(\mathrm{b}){ }^{*}(\mathrm{a}+)$ \\
\hline 5 & $20.79 \pm 1.7^{\star \star \star}(\mathrm{a})$ & $5+$ & $23.52 \pm 1.5 \mathrm{NS}(\mathrm{b}){ }^{* \star}(\mathrm{a}+)$ \\
\hline 10 & $25.27 \pm 1.5^{\star \star}(\mathrm{a})$ & $10+$ & $25.16 \pm 1.9 \mathrm{NS}(\mathrm{b}) \mathrm{NS}(\mathrm{a}+)$ \\
\hline 20 & $21.261 .0 *(a)$ & $20+$ & $22.46 \pm 1.2^{*}(\mathrm{~b}){ }^{*}(\mathrm{a}+)$ \\
\hline 40 & $19.95 \pm 0.99 \mathrm{NS}(\mathrm{a})$ & $40+$ & $23.09 \pm 0.98 *(b) N S(a+)$ \\
\hline 80 & $16.15 \pm 0.57$ & $80+$ & $18.55 \pm 0.63$ \\
\hline
\end{tabular}

Probability level: ${ }^{*} \mathrm{p}<0.05,{ }^{* *} \mathrm{p}<0.01,{ }^{* * *} \mathrm{p}<0.001 \mathrm{NS}$ : Not significant, a: Significant test between flyash and control soil T/H: Tones per hectare, FA: Flyash, DAS: Days after sowing $\mathrm{a}=$ Significant test between control and FA $\mathrm{a}+=$ Significant test between control and FA+ $\mathrm{b}=$ Significant test between FA and FA+

FA amendment in soil caused a gradual increase in $\mathrm{pH}$ from 5.7 (control) to 6.6 (80 T/H FA), however addition of Azosprillum didn't show any significant effects on $\mathrm{pH}$ level. Similarly CEC changed from 3.67(Control) to 5.8 (10 T/H FA and $\mathrm{FA}+$ ). The EC changed from 0.32 (control) to 0.47 (80 T/H FA) and 0.39 (control) to $0.53(80$ 
$\mathrm{T} / \mathrm{H} \mathrm{FA}+$ ); Organic Carbon changed from 0.80 (control) to 0.89 (80 T/H FA) and 0.84(control) to 0.90 (80 T/H FA+); Nitrogen Content changed to 0.080 (control) to 0.089 (80 $\mathrm{T} / \mathrm{H} \quad \mathrm{FA})$ and 0.084 (control) to $0.090 \quad(80 \mathrm{~T} / \mathrm{H}$ $\mathrm{FA}+$ ); Phosphate content changed to 70.02 (control) to
$98.35(80 \mathrm{~T} / \mathrm{H} \mathrm{FA})$ and 84.53 (control) to $99.02(80 \mathrm{~T} / \mathrm{H}$ $\mathrm{FA}+$ ); Potassium Content changed from 398 (control) to 697 (80 T/H FA) and 502 (control) to 540 (80 T/H FA+). Azosprillum supplemented flyash perform better in all soil properties. Results are presented in Table-10 to 16 .

Table 10: $\mathrm{pH}$ of fly ash (without and with Azosprillum) amended on Tomato field

\begin{tabular}{|c|c|c|c|c|c|}
\hline Cont. FA & $\mathbf{5 T / H}$ & $\mathbf{1 0 T / H}$ & $\mathbf{2 0 T} / \mathbf{H}$ & $\mathbf{4 0 T} / \mathbf{H}$ & $\mathbf{8 0 T} / \mathbf{H}$ \\
\hline B.C.(5.6-5.7) & $(5.6-5.7)$ & $(5.6-5.7)$ & $(5.6-5.7)$ & $(5.6-5.7)$ & $(5.6-5.7)$ \\
\hline A.C.(5.7-5.8) & $(5.7-5.8)$ & $(5.8-6.1)$ & $(6.0-6.2)$ & $(6.2-6.6)$ & $(6.2-6.6)$ \\
\hline Cont+. FA+ & $\mathbf{5 + ~ T / H}$ & $\mathbf{1 0 +} \mathbf{T} / \mathbf{H}$ & $\mathbf{2 0 +} \mathbf{T} / \mathbf{H}$ & $\mathbf{4 0 +} \mathbf{T} / \mathbf{H}$ & $\mathbf{8 0 +} \mathbf{T} / \mathbf{H}$ \\
\hline B.C.(5.8-5.9) & $(5.8-5.9)$ & $(5.8-5.9)$ & $(5.8-5.9)$ & $(5.8-5.9)$ & $(5.8-5.9)$ \\
\hline A.C.(5.8-5.9) & $(5.9-6.0)$ & $(6.0-6.2)$ & $(6.1-6.3)$ & $(6.2-6.4)$ & $(6.3-6.7)$ \\
\hline
\end{tabular}

B.C.: Before cropping, A.C.: After cropping

Table 11: Cation exchange capacity of fly ash (without and with Azosprillum) amended on Tomato field

\begin{tabular}{|c|c|c|c|}
\hline $\begin{array}{c}\text { FA Treatment } \\
\text { (T/H) }\end{array}$ & $\begin{array}{c}\text { Cation } \\
\text { exchange } \\
\text { capacity }\end{array}$ & $\begin{array}{c}\text { FA + } \\
\text { Treatment } \\
(\mathbf{T} / \mathbf{H})\end{array}$ & $\begin{array}{c}\text { Cation } \\
\text { exchange } \\
\text { capacity }\end{array}$ \\
\hline Cont. & 3.67 & Cont.+ & 3.7 \\
\hline 5 & 5.45 & $5+$ & 5.55 \\
\hline 10 & 5.8 & $10+$ & 5.81 \\
\hline 20 & 3.67 & $20+$ & 4.81 \\
\hline 40 & 3.14 & $40+$ & 4.67 \\
\hline 80 & 3.8 & $80+$ & 4.21 \\
\hline
\end{tabular}

FA: Flyash, $\mathrm{T} / \mathrm{H}$ : Tones per hectare, DAS: Days after sowing

Table 12: Electrical Conductivity of fly ash (without and with Azosprillum) amended on Tomato field

\begin{tabular}{|c|c|c|c|c|c|}
\hline Cont. FA & $\mathbf{5 T / H}$ & $\mathbf{1 0 T} / \mathbf{H}$ & $\mathbf{2 0 T} / \mathbf{H}$ & $\mathbf{4 0 T / H}$ & $\mathbf{8 0} \mathbf{T} / \mathbf{H}$ \\
\hline B.C. $(0.28-0.32)$ & $(0.28-0.32)$ & $(0.28-0.32)$ & $(0.28-0.32)$ & $(0.28-0.32)$ & $(0.28-0.32)$ \\
\hline A.C. $(0.35-0.38)$ & $(0.35-0.38)$ & $(0.39-0.40)$ & $(0.40-0.42)$ & $(0.38-0.46)$ & $(0.41-0.47)$ \\
\hline Cont+. FA+ & $\mathbf{5 +} \mathbf{T} / \mathbf{H}$ & $\mathbf{1 0 +} \mathbf{T} / \mathbf{H}$ & $\mathbf{2 0 +} \mathbf{T} / \mathbf{H}$ & $\mathbf{4 0 +} \mathbf{T} / \mathbf{H}$ & $\mathbf{8 0 +} \mathbf{T} / \mathbf{H}$ \\
\hline B.C. $(0.34-0.39)$ & $(0.34-0.39)$ & $(0.34-0.39)$ & $(0.34-0.39)$ & $(0.34-0.39)$ & $(0.34-0.39)$ \\
\hline A.C. $(0.36-0.40)$ & $(0.40-0.42)$ & $(0.39-0.41)$ & $(0.42-0.48)$ & $(0.44-0.49)$ & $(0.47-0.53)$ \\
\hline
\end{tabular}

B.C.: Before cropping, A.C.: After cropping

Table 13: Organic Carbon content (Ib/acre) of fly ash (without and with Azosprillum) amended Tomato field

\begin{tabular}{|c|c|c|c|c|c|}
\hline \multicolumn{7}{|c|}{ Organic Carbon content (Ib/acre) } \\
\hline Cont.FA & $\mathbf{5 T / H}$ & $\mathbf{1 0 T / H}$ & $\mathbf{2 0 T / H}$ & $\mathbf{4 0 T / H}$ & $\mathbf{8 0 ~ T / H}$ \\
\hline B.C. $(0.79-0.80)$ & $(0.79-0.80)$ & $(0.79-0.80)$ & $(0.79-0.80)$ & $(0.79-0.80)$ & $(0.79-0.80)$ \\
\hline A.C. $(0.81-0.82)$ & $(0.82-0.83)$ & $(0.86-0.85)$ & $(0.85-0.87)$ & $(0.86-0.88)$ & $(0.87-0.89)$ \\
\hline Cont+.FA+ & $\mathbf{5 + ~ T / H}$ & $\mathbf{1 0 +} \mathbf{T} / \mathbf{H}$ & $\mathbf{2 0 +} \mathbf{T} / \mathbf{H}$ & $\mathbf{4 0 +} \mathbf{T} / \mathbf{H}$ & $\mathbf{8 0 +} \mathbf{T} / \mathbf{H}$ \\
\hline B.C. $(0.82-0.84)$ & $(0.82-0.84)$ & $(0.82-0.84)$ & $(0.82-0.84)$ & $(0.82-0.84)$ & $(0.82-0.84)$ \\
\hline A.C. $(0.85-0.87)$ & $(0.86-0.87)$ & $(0.86-0.88)$ & $(0.87-0.89)$ & $(0.85-0.87)$ & $(0.88-0.90)$ \\
\hline
\end{tabular}

B.C.: Before cropping, A.C.: After croppin 
Table 14: Nitrogen content of fly ash (without and with Azosprillum) amended on Tomato field

\begin{tabular}{|c|c|c|c|c|c|}
\hline \multicolumn{7}{|c|}{ Nitrogen content (Ib/acre) } \\
\hline Cont.FA & $\mathbf{5 T / H}$ & $\mathbf{1 0 T / H}$ & $\mathbf{2 0 T / H}$ & $\mathbf{4 0 T / H}$ & $\mathbf{8 0 T / H}$ \\
\hline B.C.(0.079-0.080) & $(0.079-0.080)$ & $(0.079-0.080)$ & $(0.079-0.080)$ & $(0.079-0.080)$ & $(0.079-0.080)$ \\
\hline A.C.(0.081-0.082) & $(0.082-0.083)$ & $(0.086-0.085)$ & $(0.085-0.087)$ & $(0.086-0.088)$ & $(0.087-0.089)$ \\
\hline Cont+.FA+ & $\mathbf{5 + ~ T / H}$ & $\mathbf{1 0 +} \mathbf{T} / \mathbf{H}$ & $\mathbf{2 0 +} \mathbf{T} / \mathbf{H}$ & $\mathbf{4 0 +} \mathbf{T} / \mathbf{H}$ & $\mathbf{8 0 +} \mathbf{T} / \mathbf{H}$ \\
\hline B.C.(0.082-0.084) & $(0.082-0.084)$ & $(0.082-0.084)$ & $(0.082-0.084)$ & $(0.082-0.084)$ & $(0.082-0.084)$ \\
\hline A.C.(0.085-0.087) & $(0.086-0.087)$ & $(0.086-0.088)$ & $(0.087-0.089)$ & $(0.085-0.087)$ & $(0.088-0.090)$ \\
\hline
\end{tabular}

B.C.: Before cropping, A.C.: After cropping

Table 15: Phosphate content of fly ash (without and with Azosprillum) amended on Tomato field

\begin{tabular}{|c|c|c|c|c|c|}
\hline \multicolumn{7}{|c|}{ Phosphate content (Ib/acre) } \\
\hline Cont. FA & $\mathbf{5 T / H}$ & $\mathbf{1 0 T} / \mathbf{H}$ & $\mathbf{2 0 T / H}$ & $\mathbf{4 0 T / H}$ & $\mathbf{8 0} \mathbf{T} / \mathbf{H}$ \\
\hline B.C.(63.83-70.02) & $(63.83-70.02)$ & $(63.83-70.02)$ & $(63.83-70.02)$ & $(63.83-70.02)$ & $(63.83-70.02)$ \\
\hline A.C.(83.95-84.62) & $(89.7-90.98)$ & $(89.88-91.63)$ & $(90.98-92.89)$ & $(97.05-98.08)$ & $(97.55-98.35)$ \\
\hline Cont+. FA+ & $\mathbf{5 + ~ T / H}$ & $\mathbf{1 0 +} \mathbf{T} / \mathbf{H}$ & $\mathbf{2 0 +} \mathbf{T} / \mathbf{H}$ & $\mathbf{4 0 +} \mathbf{T} / \mathbf{H}$ & $\mathbf{8 0 +} \mathbf{T} / \mathbf{H}$ \\
\hline B.C.(82.52-84.53) & $(82.52-84.53)$ & $(82.52-84.53)$ & $(82.52-84.53)$ & $(82.52-84.53)$ & $(82.52-84.53)$ \\
\hline A.C.(83.12-86.49) & $(86.35-87.15)$ & $(89.70-90.80)$ & $(96.60-98.20)$ & $(96.35-98.40)$ & $(97.42-99.02)$ \\
\hline
\end{tabular}

B.C.: Before cropping, A.C.: After cropping

Table 16: Effect Influence of FA on soil elemental (in ppm) status (without and with Azosprillum).

\begin{tabular}{|c|c|c|c|c|c|c|c|c|c|c|c|c|c|c|}
\hline \multirow{2}{*}{$\begin{array}{c}\text { FA } \\
\text { Treatment } \\
(\mathrm{T} / \mathrm{H})\end{array}$} & \multicolumn{2}{|c|}{ Fe } & \multicolumn{2}{|c|}{ Mn } & \multicolumn{2}{|c|}{$\mathrm{Pb}$} & \multicolumn{2}{|c|}{ Co } & \multicolumn{2}{|c|}{$\mathrm{Na}$} & \multicolumn{2}{|c|}{$\mathrm{K}$} & \multicolumn{2}{|c|}{$\mathbf{P}$} \\
\hline & FA & $\mathrm{FA}+$ & FA & $\mathrm{FA}+$ & FA & $\mathrm{FA}+$ & FA & $\mathrm{FA}+$ & FA & $\mathrm{FA}+$ & FA & $\mathrm{FA}+$ & FA & $\mathrm{FA}+$ \\
\hline Cont. & 650 & 600 & 18.8 & 12.4 & 2.8 & 2.0 & 1.4 & 1.0 & 1180 & 1200 & 3900 & 3600 & 45.5 & 45.5 \\
\hline 5 & 720 & 703 & 45 & 36.0 & 8.8 & 8.0 & 3.2 & 2.4 & 1215 & 1005 & 4280 & 4600 & 36.9 & 45.5 \\
\hline 10 & 686 & 662 & 42.4 & 25.8 & 5.4 & 5.2 & 1.8 & 1.6 & 1180 & 1215 & 6500 & 5890 & 46.0 & 68.2 \\
\hline 20 & 795 & 722 & 31.6 & 19.8 & 5.8 & 4.4 & 1.6 & 1.0 & 900 & 950 & 7050 & 7700 & 50.3 & 90.9 \\
\hline 40 & 811 & 692 & 20.8 & 15.6 & 1.8 & 1.6 & 1.2 & 0.8 & 850 & 1005 & 8290 & 8000 & 46.8 & 68.0 \\
\hline 80 & 664 & 633 & 19.4 & 14.2 & 1.6 & 1.4 & 1.1 & 0.8 & 1050 & 1050 & 10150 & 9650 & 70.0 & 82.0 \\
\hline
\end{tabular}

Table 17: Potassium content of flyash (without and with Azosprillum) amended on Tomato field

\begin{tabular}{|c|c|c|c|c|c|}
\hline \multicolumn{7}{|c|}{ Potassium content (Ib/acre) } \\
\hline Cont. FA & $\mathbf{5 T / H}$ & $\mathbf{1 0 T} / \mathbf{H}$ & $\mathbf{2 0 T} / \mathbf{H}$ & $\mathbf{4 0 T / H}$ & $\mathbf{8 0} \mathbf{~ T / H}$ \\
\hline B.C.(388-398) & $(388-398)$ & $(388-398)$ & $(388-398)$ & $(388-398)$ & $(388-398)$ \\
\hline A.C.(390-400) & $(390-420)$ & $(410-448)$ & $(423-456)$ & $(490-560)$ & $(618-697)$ \\
\hline Cont+. FA+ & $\mathbf{5 + T / H}$ & $\mathbf{1 0 +} \mathbf{T} / \mathbf{H}$ & $\mathbf{2 0 +} \mathbf{T} / \mathbf{H}$ & $\mathbf{4 0 +} \mathbf{T} / \mathbf{H}$ & $\mathbf{8 0 +} \mathbf{T} / \mathbf{H}$ \\
\hline B.C.(410-428) & $(458-469)$ & $(444-480)$ & $(462-458)$ & $(481-499)$ & $(457-502)$ \\
\hline A.C.(423-440) & $(468-478)$ & $(456-480)$ & $(469-500)$ & $(511-532)$ & $(510-540)$ \\
\hline
\end{tabular}

B.C.: Before cropping, A.C.: After cropping 
Table 18: Effect of different concentrations of fly ash emended soil (without and with Azosprillum) on heavy metal uptake of leaves of Lycopersium esculentum C.V. S-28

\begin{tabular}{|c|c|c|c|c|c|c|c|c|}
\hline \multirow{2}{*}{$\begin{array}{c}\text { FA Treatment } \\
(\mathbf{T} / \mathbf{H})\end{array}$} & \multicolumn{8}{|c|}{ Heavy metal uptake (ppm) } \\
\cline { 2 - 9 } & \multicolumn{2}{|c|}{ Fe } & \multicolumn{2}{c|}{ Mn } & \multicolumn{2}{c|}{ Pb } & \multicolumn{2}{c|}{ Co } \\
\cline { 2 - 9 } & FA & FA+ & FA & FA+ & FA & FA+ & FA & FA+ \\
\hline Cont. & 650 & 600 & 18.8 & 12.4 & 2.8 & 2.0 & 1.4 & 1.0 \\
\hline 5 & 720 & 703 & 45 & 36.0 & 8.8 & 8.0 & 3.2 & 2.4 \\
\hline 10 & 686 & 662 & 42.4 & 25.8 & 5.4 & 5.2 & 1.8 & 1.6 \\
\hline 20 & 795 & 722 & 31.6 & 19.8 & 5.8 & 4.4 & 1.6 & 1.0 \\
\hline 40 & 811 & 692 & 20.8 & 15.6 & 1.8 & 1.6 & 1.2 & 0.8 \\
\hline 80 & 664 & 633 & 19.4 & 14.2 & 1.6 & 1.4 & 1.1 & 0.8 \\
\hline
\end{tabular}

FA: Flyash, $\mathrm{T} / \mathrm{H}$ : Tones per hectare

The Fe contents of soil remain almost unchanged due to FA amendments. Microelements $\mathrm{Mn}, \mathrm{Pb}$, Co had progressively increased due to increasing FA amendment (Table-17). Azosprillum supplementations caused lowering of the concentrations in soils. Elemental analysis of Lycopersicum esculantum $\mathrm{L}$. leaves revealed significant changes in different levels in different metals. $\mathrm{Fe}$ accumulates from 650 (Control) to $811 \mathrm{ppm}$ at $40 \mathrm{~T} / \mathrm{H}$ $\mathrm{FA} ; \mathrm{Mn}$ accumulates from 18.80 (Control) to $45.0 \mathrm{ppm}$ at $5 \mathrm{~T} / \mathrm{H} \mathrm{FA} ; \mathrm{Pb}$ accumulates from 2.80 (Control) to 8.8 $\mathrm{ppm}$ at $5 \mathrm{~T} / \mathrm{H} \mathrm{FA}$; Co accumulates from 1.4 (Control) to 3.2ppm at $5 \mathrm{~T} / \mathrm{H}$ FA due to FA amendments. Azosprillum supplementations caused an invariable decrease of each value. Surprisingly toxic elements accumulate at lower concentration of FA.

Field experiments, carried out with Lycopersicum esculantum L. grown in FA amendments, clearly indicated that growth of tomato crop was significantly increased by FA, particularly around $40 \mathrm{~T} / \mathrm{H}$ FA for Lycopersicum esculantum L. Similarly results of growth enhancements by FA have been recorded for several crops, grown in other countries (Koracak, 1995; Allen et al., 1974). Ecological studies in effects of FA contaminating terrestrial and aquatic habitats have also been well documented (Twardowska, 1990; Mishuntinand and Shilinikova, 1971). Similarly increase in growth and yield of numerous crops and vegetables like Medicago sativa, Hordeum vulgare, Zea mays, Sorghum bicolor, Echinochola crusgalli, Dacus carota, Allium cepa, Phaseolus vulgaris, Brassica oleracea (Aggarwal et al., 2009; Siddiqui and Singh, 2005) have been observed by various researchers. The presence of essential plant nutrients such as $\mathrm{K}, \mathrm{Mg}, \mathrm{S}$ and micronutrients make FA a source of important plant nutrients which influences the plant growth (Muduli et al., 2014). All these reports recorded heavy metal pollutions due to FA and accumulations of such recalcitrant ions in all the crops observed. In addition to plant growth there are improvements of soil physical properties, EC, CEC due to FA amendment. Increase of EC values due to FA could suggest that the binding of metal ions occurred readily to soil particles, causing the eventual availability/ entry of metal nutrients to growing plants. Increase in growth and yield of Lycopersicum esculantum in FA amended soil was due to ready supply of some excess plant nutrients without any interference of the higher $\mathrm{pH}$ range to soil plant relationship, as exemplified by Calmano et al. (1993). Moreover, Calmona et al. (1993) had shown the binding forms of heavy metals $(\mathrm{Zn}, \mathrm{Pb}$, $\mathrm{Cu}$ and $\mathrm{Cd}$ ) change significantly by periodic redox changes in a low-buffered sediment. Further, within limits of $10 \mathrm{~T} / \mathrm{H} \mathrm{FA}$, an amendment of tropical tomato soil in the water logging condition should be a boon. This finding is encouraging for an agro-friendly disposal of this potential multiple pollutants in tomato cultivation and soil fertilization as well. Comparative increase in $\mathrm{K}, \mathrm{P}$ and Fe contents in soil by FA was found to have additive effect on growth and yield of tomato.

The observed decline in yield at higher FA levels may be linked to metal toxicity caused by accumulation of $\mathrm{Mn}, \mathrm{Pb}, \mathrm{Co}, \mathrm{Fe}$ in plant tissues as in other crops (Korcak, 1995). The toxic elements were in concentration of low level in the presently used FA level. In the present study reported heavy metal level in the leaves of tomato plant was not about threshold concentrations determined to that of crucifers (Davies, 1992).

Based on the data obtained it could be concluded that the soil amended at 20 to $40 \mathrm{~T} / \mathrm{H}$ not only improved the physical properties of the soil but also contributed to the better growth and yield of tomato. Simultaneously contamination of heavy metal ions was found progressive with doses of FA remaining below the threshold levels. Azosprillum supplementations at the FA level used in soil had additive effects concerning all growth parameters and deductive effects on accumulation of toxic elements in soil and plant were recorded. Keeping in view of the heavy metal toxicity FA should be used with biological reagents for better yield and lesser toxicity. 
Odisha, India. Finally we are thankful to Dr. G. Nahak for helping in computer work without which preparation of this manuscript would not have been possible.

\section{REFERENCES}

Aggarwal, S., G.R. Singh and B.R. Yadav, 2009. Utilization of flyash for crop production; Effect on the growth of wheat and sorghum crops and soil properties. Journal of Agricultural physics, 9: 20-23.

Alef, K. and P. Nannipieri, 1995. Methods in Applied Soil Microbiology and Biochemistry. London: Academic Press, p. 88-91.

Allen, S.E., H.M. Grimshaw, J.A. Kensin and C. Quarmby, 1974. Chemical analysis of ecological materials. Blackwell Scientific, Oxford, p. 569.

Bremner, J.M. and D.S. Jenkinson: Determination of organic carbon in soil. I. Oxidation by dichromate of organic matter in soil and plant materials. J. Soil Sci., 11: 394-402.

Calmano, W, J. Hong, U. Forstner and J. Hong, 1993. Binding and mobilization of heavy metals in contaminated sediments affected by $\mathrm{pH}$ and redox potential. Water Sci Technol., 28: 53-58.

Camberato, J.J., E.D. Vance and A.V. Someshwar, 1997. Composition and land application of paper manufacturing residuals. In: Rechcigl J, MacKinnon $\mathrm{H}$ (eds) agricultural uses of byproducts and wastes. ACS, Washington DC, p. 185-203.

Davies, B.E., 1992. Inter-relationships between soil properties and the uptake of cadmium, copper, lead and zinc from contaminated soils by radish (Raohanus sativus L.). Water Air Soil Pollut., 63: 33-342.

Fail, J.L. and Z.S. Wochok, 1977. Soybean growth on flyash amended strip mine spoils. Plants and soil, 48: 437-484.

Fytianos, K., G. Katsianis, P. Triantafyllou and G. Zachariadis, 2001. Accumulation of Heavy Metals in Vegetables Grown in an Industrial Area in Relation to Soil. Bulletin of Environmental Contamination and Toxicology, 67(3): 423430.

Harper, D.J., G.F. Fileman, P.V. May and J.E. Portman, 1989. The analysis of trace metals in marine and other sample. Aquatic environment orotocols: Analytical methods. MAFF Direct fish. Research. Lowestroft, UK, p. 450.

Harris, R., M. Cremers, Ebinger and B. Bluhm, 2004. Determination of Nitrogen in Sand Using Laser Induced Breakdown Spectroscopy. Appl. Spectrosc., 58: 770-775.

Khan, M. R. and M. W. Khan, 1996. The effect of fly-ash on plant growth and yield of tomato. Environ. Pollut., 92: 105111.

Koracak R.F., 1995. Utilization of coal combustion byproduct agriculture and horticulture. In: Agricultural utilization of urban and industrial by-products. ASA Spec. Publ. 58.
(Eds. D.L. Karlen et al.) ASA. CSSA, Madison, Wi, p. 107130.

Miller, D.M., W.P. Miller, S. Dudka and M.E. Sumner, 2000. Characterization of industrial by-products. In: Dick WA et al (eds) Land Application of Agricultural, Industrial, and Municipal By-Products. SSSA Book series: 6, SSSA, Madisoon, W.I., p.107-119.

Mishra, L.C. and K.N. Shukla, 1986. Effect of flyash Deposition on growth metabolism and dry matter production of maize and soybean. Environment pollution (Series A) 42: 1-13.

Mishra, M, R.K. Sahu and R.N. Padhy, 2005. Effect of vermicomposted municipal solid waste on growth, yield, and heavy metal contents of rice (Oryza sativa). Fresenius Environ Bull, 14: 584-590.

Mishra, M., R. K. Sahu, S. K. Sahu, and R. N. Padhy, 2009. Growth, yield and elements content of wheat (Triticum aestivum) grown in composted municipal solid wastes amended soil. Environ. Develop. Sustain., 11: 115-126.

Mishuntinand, E.N. and V.K. Shilinikova, 1971. Biological fixation of atmospheric nitrogen. McMillan, London, p. 215.

Muduli, S.D., B.D. Nayak, N.K. Dhal and B.K. Mishra, 2014. Atmospheric $\mathrm{CO}_{2}$ sequestration through Mineral carbonation of flyash. Greener Journal of Physical Sciences, 4(1): 273-278.

Shaw, P.J.A., 1992. A preliminary study of successional changes in vegetation and development on unamended flyash (FA) in Southern England. J. Appl. Ecol., 29: 728736.

Siddiqui, Z. A. and L.P. Singh, 2005. Effects of flyash and soil micro-organisms on plant growth, photosynthetic pigments and leaf blight of wheat. Journal of plant Diseases and Protection, 112 (2): 146-155.

Sumner, M.E. and W.P. Miller, 1996. Cation exchange capacity, and exchange coefficients. In: D.L. Sparks (ed.) Methods of soil analysis. Part 2: Chemical properties (3rd ed.). ASA, SSSA, CSSA, Madison, WI.

Thomas, G.W., 1982. Exchangeable cations. In: A.L. Page et al. (eds.), Methods of Soil Analysis, Part 2, 2nd ed. Agron. Monogr. 9. ASA and SSSA, Madison, WI. p. 159-165.

Walkly, Y.A. and I.A. Black, 1934. An examination of digestion method for determining soil organic matter and a proposed modification of the chromic acid titration method. Soil Sci., 37: 29-38.

Wardowska, I.T., 1990. Buffering capacity of coal mines spoils and flyash as a factor in the protection of the aquatic environment. The Sci. Total Environ., 91:177-189.

Warncke, D. and J.R. Brown, 1982. Potassium and other basic cations. In: J.R. Brown (ed.), Recommended Chemical Soil Test Procedures for the North Central Region. (Revised.) Missouri Agr. Exp. Sta. SB1001. Columbia, MO. p. 31-33. 